\title{
Spectral analysis of mid-IR excesses of central stars of planetary nebulae
}

\author{
Jana Bilikova ${ }^{1}$, You-Hua Chu ${ }^{1}$, Kate Y.L. $\mathrm{Su}^{2}$, Robert Gruendl ${ }^{1}$, et al. \\ ${ }^{1}$ Dept. of Astronomy, University of Illinois, \\ 1002 West Green Street, Urbana, Illinois, 61801, USA \\ email: jbiliko2@illinois.edu \\ ${ }^{2}$ Stewart Observatory, University of Arizona, \\ Tucson, AZ 85721, USA \\ email: ksu@as .arizona.edu
}

\begin{abstract}
In our Spitzer $24 \mu \mathrm{m}$ survey of hot white dwarfs (WDs) we found 9 WDs with IR excesses, 7 of them are still central stars of planetary nebulae (CSPNs). We have thus carried out a Spitzer archival survey of CSPNs, and found additional objects with IR excesses. To date, a total of 13 CSPNs show IR excesses from Spitzer observations. These mid-IR excesses are indicative of the presence of circumstellar dust, which could be produced by collisions or disruption of sub-planetary objects. To further assess the nature of these IR excesses, we have obtained Spitzer IRS, Gemini NIRI and Michelle, and KPNO 4m echelle spectra of these objects. In this poster, we summarize the spectroscopic observations and discuss the nature of these IR excesses.
\end{abstract}

Keywords. planetary nebulae: general, infrared: stars, circumstellar matter

\section{Introduction}

Spitzer Space Telescope MIPS 24 and $70 \mu \mathrm{m}$ observations of Helix Nebula's hot $\left(\mathrm{T}_{\text {eff }}\right.$ $\sim 110,000 \mathrm{~K}) \mathrm{CSPN}$ revealed a compact source coincident with the central WD. Its followup Spitzer IRS spectrum confirmed that the mid-IR emission originates from a 90-130 K dust continuum with an emitting area of 4-40 $\mathrm{AU}^{2}$, located between 40 and $100 \mathrm{AU}$ from the CSPN. Such location corresponds to that of the Kuiper Belt in our Solar System, and the dust disk was suggested to originate from collisions among Kuiper Belt-like objects, dynamically rejuvenated in the AGB and post-AGB evolutionary stages ( $\mathrm{Su}$ et al. 2007).

\section{CSPNs with IR Excesses}

To find more dust disks similar to that of the Helix Nebula's CSPN, we have surveyed 71 hot WDs for excess $24 \mu \mathrm{m}$ emission with Spitzer's MIPS camera. We have found 9 cases of IR excess, in 7 cases the star is still in PN (Chu et al.2011). We have also searched the Spitzer archive for other incidences of CSPNs with IR excesses, and found 19 CSPNs with clear/likely IR excesses. Among these, eight are WC stars, one is a nova, and two have companions that can account for the observed excess emission. For the remaining cases, a dust disk is required to explain the IR excess. To assess the nature of these IR excesses, we have obtained Spitzer IRS, Gemini NIRI and Michelle spectra of some of these objects. The CSPNs with dust disks, and the basic characteristics, nature, and models of the excess emission are summarized in Table 1.

Figure 1 shows multi-wavelength images, SED, IRS spectrum, and dust disk model of an example CSPN with IR excess at $24 \mu \mathrm{m}$, Sh 2-216. The excess emission originates 
Table 1. Hot White Dwarfs and CSPNs with IR Excesses

\begin{tabular}{|c|c|c|c|c|c|c|c|c|c|}
\hline $\begin{array}{l}\text { WD } \\
\text { Name }\end{array}$ & $\begin{array}{l}\text { Spec } \\
\text { Type }\end{array}$ & $\begin{array}{l}T_{\text {eff }} \\
(\mathrm{K})\end{array}$ & $\begin{array}{c}\text { JHK } \\
\text { excess }\end{array}$ & $\begin{array}{l}\text { IRAC } \\
\text { excess }\end{array}$ & $\begin{array}{l}24 \mu \mathrm{m} \\
\text { excess }\end{array}$ & $\begin{array}{l}\text { Excess } \\
\text { Nature }^{a}\end{array}$ & $\begin{array}{c}\text { Known } \\
\text { Comp }\end{array}$ & $\begin{array}{r}T_{\mathrm{dust}} \\
(\mathrm{K})\end{array}$ & $L_{\mathrm{IR}} / L_{*}$ \\
\hline CSPN Abell 21 & PG1159 & 130,000 & & & $\checkmark$ & & & 150 & $1.7 \times 10^{-5}$ \\
\hline CSPN DeHt 5 & DAO .86 & 76,500 & & $8 \mu \mathrm{m}$ & $\checkmark$ & & & 190 & $5.9 \times 10^{-5}$ \\
\hline CSPN EGB 1 & DA.34 & 150,000 & & $8 \mu \mathrm{m}$ & $\checkmark$ & $\mathrm{C}$ & & 190 & $1.3 \times 10^{-5}$ \\
\hline CSPN EGB 6 & DA.46 & 110,000 & $\checkmark$ & $\checkmark$ & $\checkmark$ & $\mathrm{C}^{b}$ & $\checkmark$ & $150+500$ & $4.6 \times 10^{-4}$ \\
\hline CSPN K 1-22 & $\mathrm{CSPN}$ & 141,000 & $\checkmark$ & $\checkmark$ & $\checkmark$ & $\mathrm{C}$ & $\checkmark$ & $150+700$ & $1.1 \times 10^{-4}$ \\
\hline CSPN NGC 2346 & $\mathrm{CSPN}$ & 100,000 & $\checkmark$ & $\checkmark$ & $\checkmark$ & $\mathrm{C}, \mathrm{E}$ & $\checkmark$ & $250+1000$ & $2.3 \times 10^{-2}$ \\
\hline CSPN NGC 2438 & $\mathrm{CSPN}$ & 114,000 & & $\checkmark$ & $\checkmark$ & $\mathrm{C}, \mathrm{E}$ & & $150+1100$ & $4.7 \times 10^{-4}$ \\
\hline CSPN NGC 6804 & CSPN & 86,000 & $\checkmark$ & $\checkmark$ & $\checkmark$ & $\mathrm{C}, \mathrm{E}$ & & $200+630+1500$ & $1.0 \times 10^{-3}$ \\
\hline CSPN NGC 6853 & $\mathrm{DAO} .46$ & 108,600 & & $8 \mu \mathrm{m}$ & $\checkmark$ & & & 150 & $2.4 \times 10^{-4}$ \\
\hline CSPN NGC 7139 & CSPN & 125,000 & & $\checkmark$ & & $\mathrm{C}$ & & 1500 & $6.3 \times 10^{-4}$ \\
\hline CSPN NGC 7293 & DAO. 49 & 110,000 & & $8 \mu \mathrm{m}$ & $\checkmark$ & $\mathrm{C}$ & & 120 & $2.5 \times 10^{-4}$ \\
\hline CSPN Sh $2-216$ & DA. 61 & 83,000 & & & $\checkmark$ & $\mathrm{C}$ & & 150 & $3.7 \times 10^{-6}$ \\
\hline CSPN Sh $2-188$ & DAO. 49 & 102,000 & & $\checkmark$ & $\checkmark$ & & & $150+900$ & $6.6 \times 10^{-5}$ \\
\hline
\end{tabular}

${ }^{a} \mathrm{C}$ stands for continuum, E for emission lines

${ }^{b} \mathrm{~A}$ Compact nebulosity is coincident with WD's companion
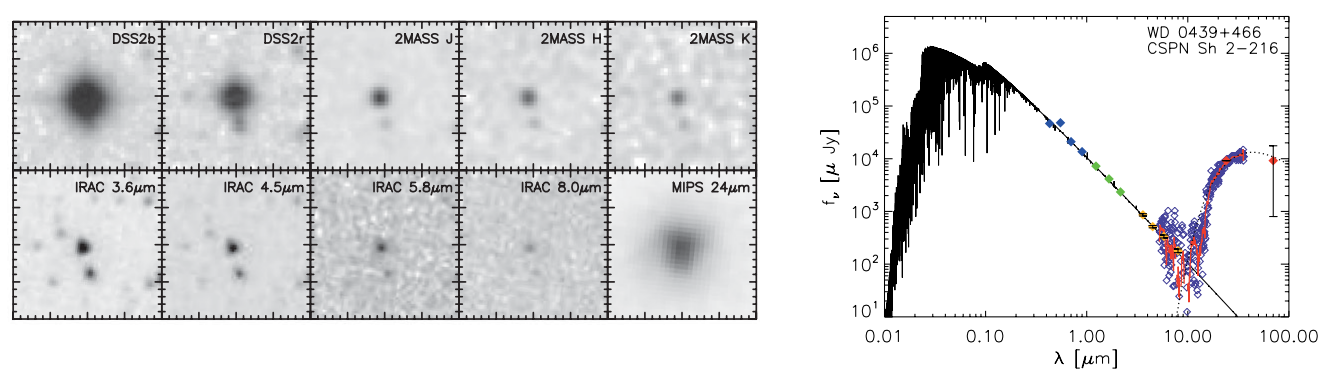

Figure 1. Images (left) and the SED (right) of CSPN Sh2-216. The Spitzer IRS spectrum is shown in the SED as open diamonds, and the smoothed spectrum is shown in thick line. The spectrum is dominated by featureless continuum emission, which starts rising at $\sim 10 \mu \mathrm{m}$. The dust model is shown as a dotted curve.

from a $T \sim 150 \mathrm{~K}$ dust continuum located between $\sim 30-35$ AU from the CSPN, with an emitting area of $\sim 0.5 \mathrm{AU}^{2}$, and a mass of $\sim 0.001$ Earth mass.

\section{Possible Origins of IR excesses}

Apart from collisions among planetesimals, other origins for the observed dust disks need to be considered. Stable Keplerian-rotating dust disks are common among binary post-AGB stars (de Ruyter et al. 2006). The CSPNs have just evolved past the post-AGB stage, and some of our targets have known binary companions, or have SEDs that do not eliminate the possibility of a companion; it is thus possible that dust disks around CSPNs may be related to those around post-AGB stars. However, it is not yet known how and whether the post-AGB binaries with dust disks evolve into the PN stage. Further observational and theoretical studies are necessary to distinguish between the two origins.

\section{References}

Chu, Y. H., Su, K. Y. L., Bilikova, J., et al. 2011, AJ, 142, 75

$\mathrm{Su}, \mathrm{K}$. Y. L., Chu, Y. H., Rieke, G. H., et al. 2007, ApJL, 28, 490

De Ruyter, S., van Winckel, H., Maas, T., et al. 2006, A\&A 448, 641 